\title{
Research on Design Method of Interactive Interface of Industrial Robot Teaching Device
}

\author{
Juan Guo \\ Xiamen University Tan Kah Kee College and VIBOT \\ Industrial Robot Innovation Laboratory \\ Xiamen University Tan Kah Kee College \\ Xiamen, China \\ juan8991@126.com
}

Yijuan Ji

Xiamen University Tan Kah Kee College and VIBOT Industrial Robot Innovation Laboratory Xiamen University Tan Kah Kee College

Xiamen, China

349565556@qq.com

\author{
Yajun Wei \\ Xiamen University Tan Kah Kee College and VIBOT \\ Industrial Robot Innovation Laboratory \\ Xiamen University Tan Kah Kee College \\ Xiamen, China \\ 18505093298@163.com
}

Yichen Zhu

Xiamen University Tan Kah Kee College and VIBOT

Industrial Robot Innovation Laboratory

Xiamen University Tan Kah Kee College

Xiamen, China

519055560@qq.com

\author{
Guozhi Luo \\ Xiamen University Tan Kah Kee College and VIBOT Industrial Robot Innovation Laboratory \\ Xiamen University Tan Kah Kee College \\ Xiamen, China \\ 384786368@qq.com
}

\begin{abstract}
This paper discussed the basic concepts, topic selection principles, and the assessment methods of project teaching. The application process of CDIO teaching method in project teaching is introduced according to the steps of "Conceive - Design - Implement - Operate", taking the electric vehicle (EV) project training in the smart grid technical scope for example. The project achievements reflect the effect of CDIO project teaching in improving the practice innovation ability of postgraduates majoring in electrical engineering. It is pointed out that the topic of project teaching must be selected from the perspective of engineering and exploratory, the process is independent and guaranteed, and the achievements are observable and assessable. Compared with the traditional teaching mode, project teaching pays more attention to training students' self-learning ability and practice innovation ability, which helps to cultivate postgraduates with creative thinking and creative spirit.
\end{abstract}

Keywords-Industrial robot; Teaching device; Interactive interface; Method

\section{INTRODUCTION}

With the development of industrial automation and intelligentialize, the field of application of industrial robots is gradually expanding [1]. Therefore the design and research of teaching device, which is the control and design terminal of industrial robots, is particularly important. However, the the interactive interface design experience optimization of the teaching device is often overlooked. The early teaching interactive experience is direct teaching, that is manually move the robot arm to teach the robot and the interactive way through the mechanical teaching box and the joystick to complete. So the teaching interactive experience is very poor and single. With the development of the teaching device, the interactive process evolved to be "operator - teaching devicecontroller-robot body". The easy operation of the teaching device and the easy-to-use interactive experience became more and more obvious. With the increase of the robot's function and intelligence, the function of the industrial robot teaching device has gradually changed from the original manual debugging to the intelligent development trend of the machine's selfrecording. Under such a circumstance, the interactive experience design method of industrial robot teaching device has a strong social application value.

\section{TEACHING DEVICE INTERACTIVE INTERFACE RESEARCH STATUS ANALYSIS}

At present the most research of industrial robot teaching device focuses on the operation research of mechanical precision and less from the user experience .With the future depth development of Industry 4.0 and the more intelligent conversion of industrial robots, the traditional interactive user experience is unable to meet the experience requirements of existing practitioners. At present the most popular robot control systems (teaching device) in the market place too much 
emphasis on the realization of technical functions and ignore the human-computer interaction experience. In actual operation interactive experience lacks humanity and many operation steps do not conform to the human inertial thinking and logic. This requires a thick user manual for searching and learning to use. As many industrial robots are approaching the same function today, the problem to be solved in this paper is to study an interface design method to simplify the teaching device interface operation, reduce the learning cost, and improve the work and study efficiency of industrial robots.

\section{A. Foreign Teaching device Interactive Interface Design Status}

In this paper the studies and generalization of the currently universal robot teaching device (TABLE I ) are summarized and the common design problems of the interaction interfaces of the existing industrial robot teaching device are summarized.
- Operation there are too many buttons on the physical interface and touch screen interface. It is not convenient for employees to remember and learn the operation.

- Interface lacks modular design, the interface tree structure is too complex and the directory is very diverse.

- The use of information graphics is less, and the developer-based interface is original. It does not include graphical interactive design of information and does not simplify the related information.

- The interactive interface of the teaching device is dominated by gray and black, so the visual guidance of color is lacked. It lacks visually efficient information transmission in a noisy industrial environment, and lacks specification of models through color difference.

TABLE I. FOREIGN TEACHING DEVICE INTERFACE USER RESEARCH ANALYSIS TABLE

\begin{tabular}{|c|c|c|}
\hline Brands & Interface Style & Functional Analysis \\
\hline Japan NACHI & 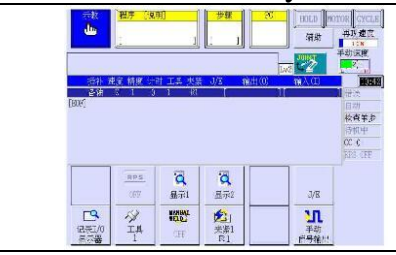 & $\begin{array}{l}\text { 1、 High degree of system interface } \\
\text { integration. } \\
\text { 2、 More physical keys. } \\
\text { 3、 Very difficult to learn. } \\
\text { 4、 Interface is not easy to understand. }\end{array}$ \\
\hline German KUKA & 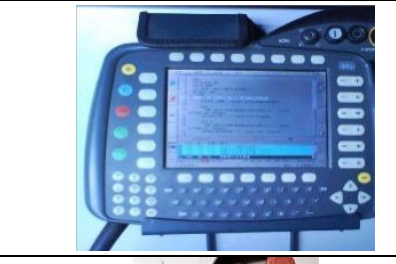 & $\begin{array}{c}\text { 1、 Most of the operating specifications and } \\
\text { interface logic. } \\
\text { 2、 The operating experience is poor and } \\
\text { learning is difficult. } \\
\text { 3、 Lack of primary and secondary } \\
\text { information. }\end{array}$ \\
\hline Italian Kemar & m & $\begin{array}{l}\text { 1、 Use a large number of physical buttons to } \\
\text { control. } \\
\text { 2、 There are few visual graphics and the } \\
\text { interface operation is not intuitive. }\end{array}$ \\
\hline Swedish ABB & 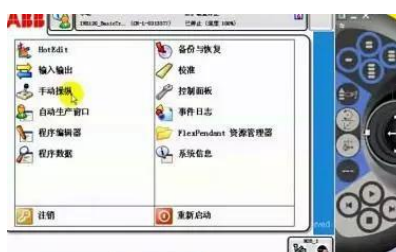 & $\begin{array}{l}\text { 1. The operation is relatively simple and the } \\
\text { interface is simple. } \\
\text { 2、 Single design style, hierarchical distinction } \\
\text { is not obvious. }\end{array}$ \\
\hline Austria keba & 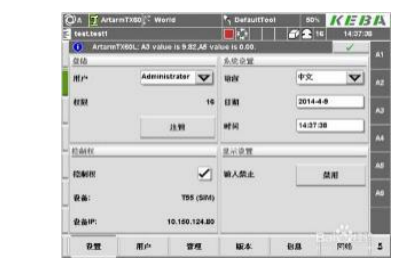 & $\begin{array}{l}\text { 1、 The distinction is very clear, the function } \\
\text { classification is clear, and the operation is } \\
\text { simple. } \\
\text { 2、 The human-computer interaction } \\
\text { experience is poor, the interface color is old, } \\
\text { and the execution efficiency is low. }\end{array}$ \\
\hline
\end{tabular}




\section{B. Domestic teaching device interface interactive interface design}

There are few innovations on the current market for selling teaching device in China. Most of them imitate the world's best-selling products (such as KUKA and ABB keba). The operating logic and interface style are similar to the teach pendant brands such as Japan and Germany, so their

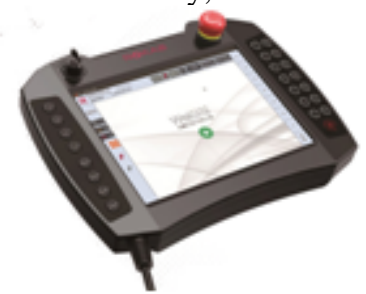

(a) Rokae (In the study) advantages and disadvantages are the same. Of course, some companies currently have some innovations in researching the teaching device. However, all of them are in the R\&D phase. The interface code and interface logic styles are classified as confidential. The following pictures are found through reference to the information documents and are for reference only (Fig. 1).

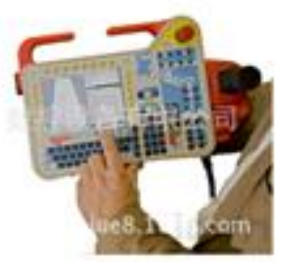

(b) Shanghai Shiyu

Fig. 1. Comparison of home-made teaching device interface

\section{DESIGNING METHOD ON INDUSTRIAL ROBOTS TEACHING DEVICE PHYSICAL INTERFACE STANDARDIZATION}

The design of the physical interaction interface is based on the hardware development of the industrial robot teaching device. Component layout, wiring methods, screen unity, and design specifications are all guarantees for the standardized interaction design of the teaching device. Especially in the industrial 4.0 intelligent industry era, on the position of hardware design, the fixed and standardized operation area can help practitioners save a lot of learning time, and improve the efficiency of production capacity through design means.

The robot teaching program developed by the laboratory adopts the MVC mechanism. The design logic is Model - View - The controller is a relatively common design pattern. This model contains three important design objects: the model provides the business logic, the view is the display of the model on the screen, and the controller is used to manage the user interaction with the view. The separation of data users and interactive interfaces can be achieved, which improves the maintainability, portability, and extensibility of the teach pendant interaction design. Through the working principle and logic research on teaching equipment, the physical logic of the existing teaching equipment is classified, extra physical keys are deleted, and the remaining physical keys are placed on the left and right sides of the fingers to avoid moving back and forth. And the adverse interaction experience of dense keys is avoided. Therefore, the positions of certain physical buttons can be fixed, and the specification and unified design can avoid frequent replacement of the positions of the physical buttons and the touch screen buttons of the multi-standard teaching equipment, resulting in waste of learning time and causing habitual misoperation. Let the interactive design of teaching equipment form a certain physical standardization and enhance the humanized design of the interactive interface.
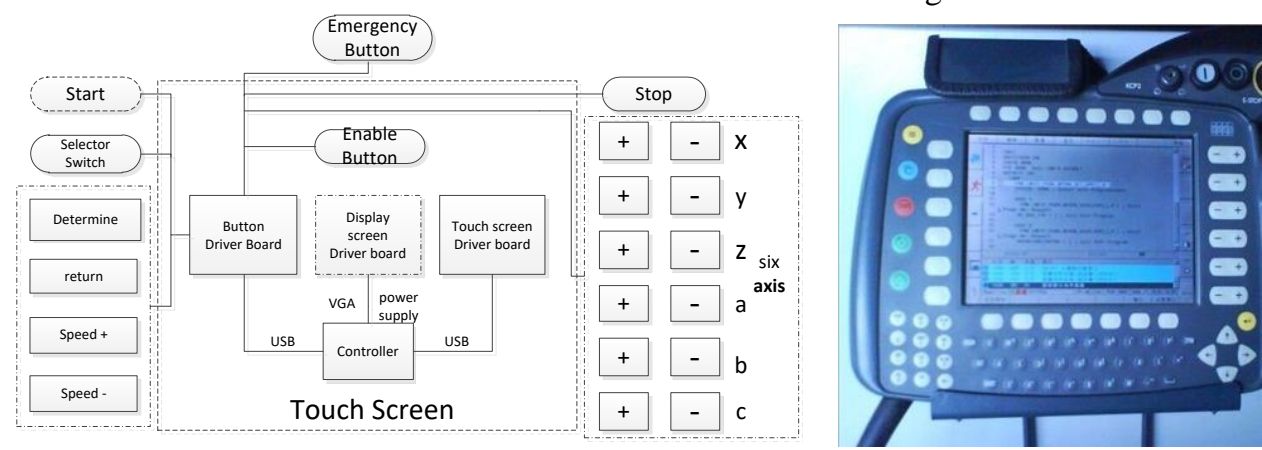

Fig. 2. Comparison of physical interface simplification of self-developed teaching device and German KUKA teaching device

The original KUKA is a brand that is currently used by the teaching device. There are too many buttons on the interface and touch screen which is inconvenient for the memory and learning of the operating personnel. Therefore, the new interface design is simplified. Some of the physical buttons are integrated into the virtual buttons, retaining the commonly used physical buttons, such as data addition and subtraction, speed buttons, emergency stop, etc. All other modules are integrated into the virtual interface. (Fig. 2)

\section{INDUSTRIAL ROBOT TEACHING DEVICE INTERACTIVE INTERFACE INFORMATION ILLUSTRATION MODULAR DESIGN METHOD}

The interface design of the existing industrial robot teach pendant is mainly physical buttons, and some of the interface design combines physical buttons and touch screen buttons. However, there is still a lack of functional integration and modular design [2]. Integrating the function information of the 
existing teaching device to form a simple and multi-module interface interaction design can not only improve the standardization of the UI interface design, but also reduce the repeated function codes of the interface development. There are many buttons in the existing teaching equipment, the level of interaction is not clear, the return operation is difficult, the experience of human-computer interaction is poor, and the difficulty in learning is large. "Fig. 3" is a comparison of the design of the keba and the lab's own R\&D interface.
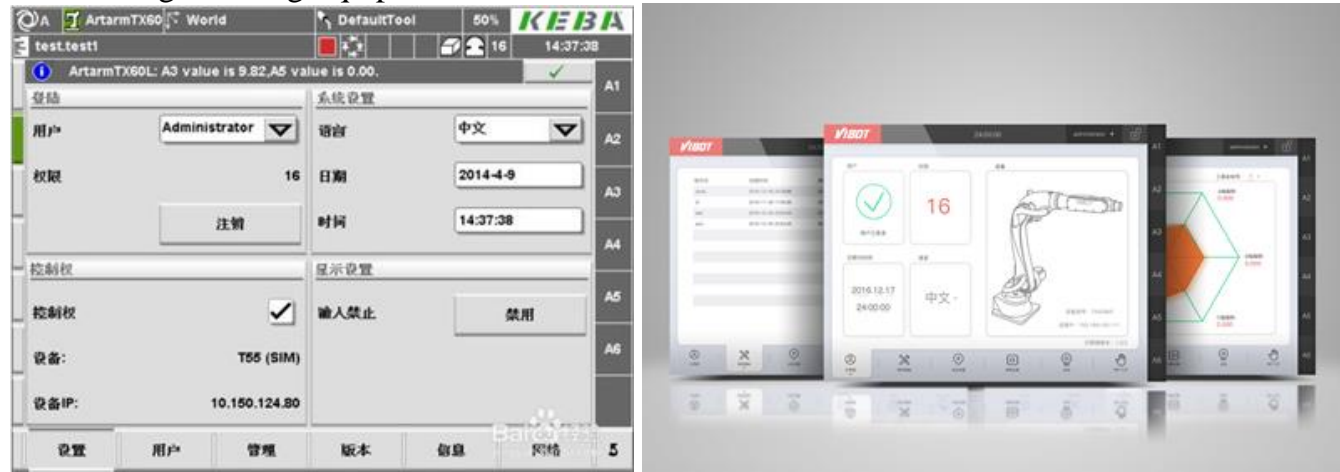

Fig. 3. Self-developed teaching device interface information diagram Modular design interface and Austria keba teaching device interface comparison chart

After analysing the actual production operations of a large number of similar products and enterprises, this paper differentiates the existing teaching device interface hierarchy and builds an architecture from the time dimension. Simplify the multi-level operation, try to make the same module operation can occur in the same action and time, sort it out and categorize, simplify the return step, make the operation smooth transition. The sequence of operations that occur at the same time is placed at the same level, making the interface more intuitive. The modules of the touch screen operation interface of the industrial robot teaching pendant are summarized into six modules, which are user technology, program editing, main interface, coordinate setting, monitoring. The information of these six modules can form a logical relationship in parallel and can be adjusted according to the needs of the user's important grades. Through the user's behavioral habits, the 6 large module interface displacements are integrated and developed, which avoids multiple repeated interface development when the teaching device function is upgraded, which greatly improves the working efficiency of the interactive interface design of the industrial robot teaching pendant [5].

The modular integrated design method will classify the functions that belong to the same category and do modular processing, focusing on the visual center of each page of the interface, enabling the viewer to quickly grasp the functional backbone and the center at the first sight. This can be used to distinguish the same type of teaching device, and solve the problem of the interactive interface clutter and catalog. Combing operations logic and architecture, simplifying unnecessary operation steps, originally requiring multiple steps to achieve operations, can be simplified one or two steps to complete operations. In a production-oriented business environment, the key steps are: Streamlining is particularly important. Modular integration simplifies the complex operation interface, provides a basis for the standardized teach pendant interface design, facilitates the subsequent upgrade and development of the robot teach pendant interactive interface, and avoids the same functional interface design multiple times.
Solved the messy information of teaching interface, too many layers, and the problem of poor interactive learning experience.

\section{INDUSTRIAL Robot RoBot TEACHING DEVICE INTERACTIVE INFORMATION VISUAL SIMPLIFIED DESIGN METHOD}

\section{A. Simplify design method of information icon of main control interface}

At present, the interface of the mainstream industrial robot teaching pendant still lacks good recognition in the design of the interface information charts, diagrams, and icons. The interface design of the main function partition of the Kawasaki teach pendant's main interface still follows the earliest windows logo design. The degree of recognition and memory are all poor, the size of the layout of the interface buttons is small, and the touch screen operation experience is poor. [3]

The main control interface has six major module functions. It uses graphical linear icons to identify the graphics. The graphics are concise and clear. The graphic and text overlay interface design allows the operator to see at a glance. In addition, the touch-screen buttons are enlarged to avoid accidental mis-clicking of the icons, thereby improving the friendliness of the interface operations.

\section{B. Secondary Interface Information Graphics Simplified Design Method}

The program programming and parameter setting interface of German KUKA teach pendant is based on the traditional code type interface. The lack of graphical information display makes it difficult for users to understand and learn [4]. In the design of the interactive interface of the industrial robot teaching device, it is necessary to introduce the graphic design method of the information graphic. Because of the large amount of programming and parameter management, the graphical interface design of the information can help the operator to quickly understand and monitor the robot's working condition. Through the informatization of different charts, diagrams, and data, the monitoring function of the teach 
pendant is improved. When we developed the teaching interface design, we converted data into a graphic display, while retaining the display of accurate data and allowing operators to see changes in data in a short time. On the important data of the robot, we have designed a global display to reduce the use of time spent on data viewing and improving the execution efficiency. The following figures 4and 5 show the interface of the existing teaching device. Compared with the self-developed graphical information interface, the interface after the design is illustrated, the information representation is clearer, and the user can quickly access the interface, and understanding the relevant parameters of the robot. It solves the problems of the teach pendant interface mentioned above.

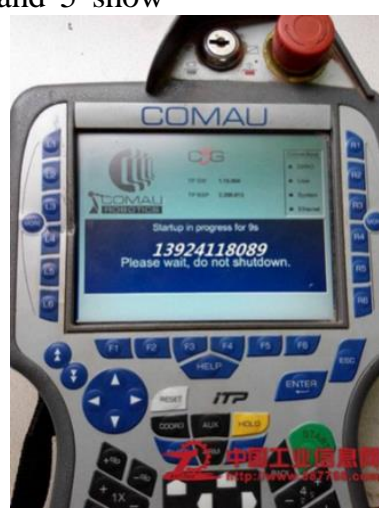

Fig. 4. Italian Comau Teaching Device Operating System

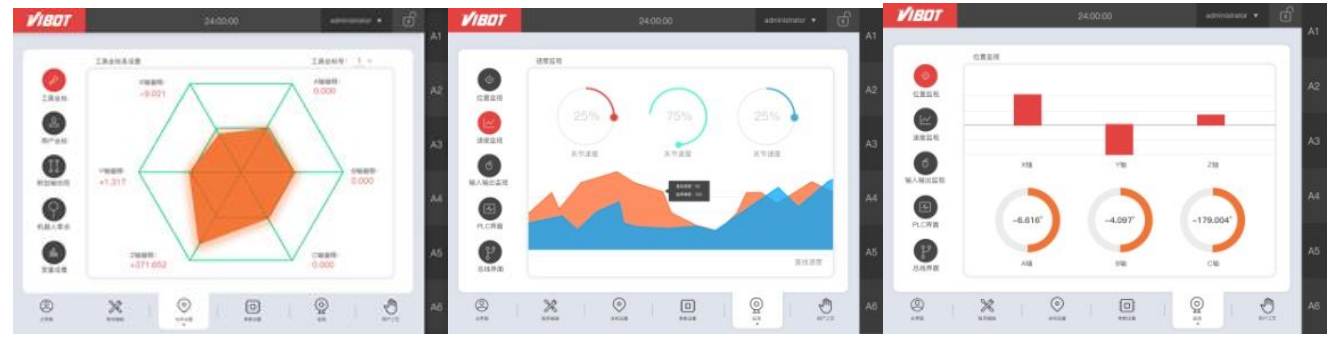

Fig. 5. Teaching device secondary interface information graphical interface design

\section{TEACHING DEVICE COLOR IDENTIFICATION MODEL STANDARDIZED INTERACTION EXPERIENCE DESIGN METHOD}

Industrial robots have a wide range of types and models, and the teaching device can be applied to the control of various robots. Therefore, the teaching device requires a unified logic specification design, otherwise it will greatly increase the cost of learning. As shown in "Fig. 1", the current teaching interface design popular in the world has a single color, and there is no color classification design in terms of color recognition. In an industrialized factory environment, sound way transmission of information is limited by noise, so the transmission of color and vision becomes the primary means of information transmission for the teaching device. The clearness of the colors facilitates visual identification and ease of operation of the interactive interface [6].
The user group of the industrial robot teaching device is mainly divided into three types: $R \& D$ engineers, maintenance engineers, and general operating users. For an industrial robot teaching device, there is necessary for a design method to quickly identify the robot type. In this paper, the Color System Color Circle of the Japan Institute of Color Research (Fig.6) is used to distinguish the type and model of the robot by using the color of the teaching device interface. The function of the robot is numbered by the Japanese color ring system. Then corresponding to the parameters of the color circle, the corresponding color design library is establish, and finally the function of different robot arms can be identified through color. At the same time solved the problem that the color specification in the teaching pendant is not perfect and the visual guidance is imperfect. This article establishes a complete visual color recognition system in the process of developing the color of the teaching device, which will be conducive to the development and upgrade of the teaching device in the future. 


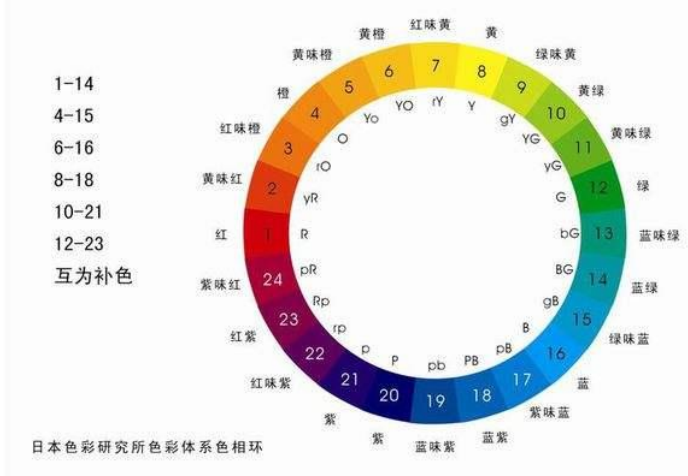

Fig. 6. Japanese color system color circle

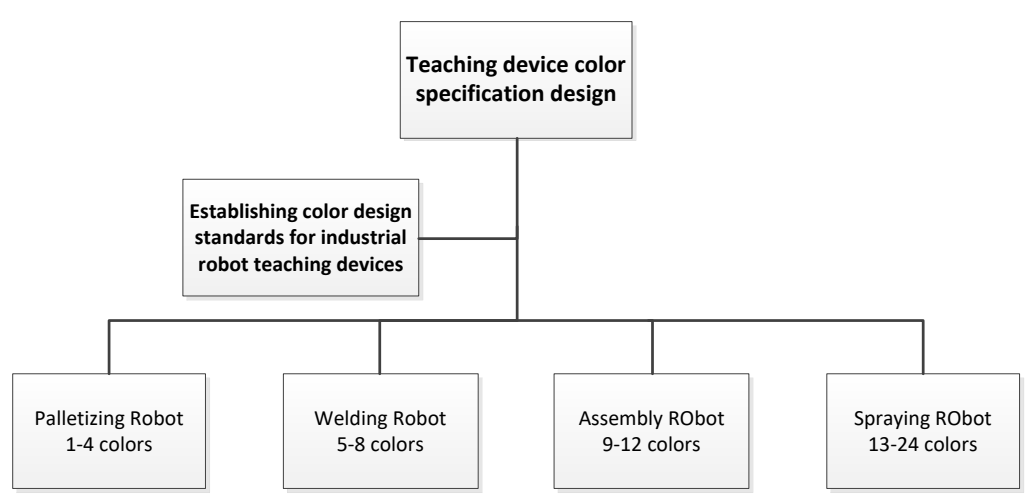

Fig. 7. Industrial robot teaching device interface design color specification

\section{CONCLUSION}

With the continuous deepening of the application of industrial robots, as the important control and debugging system terminal, the value of research on teaching device has become increasingly prominent. This article summarizes four kinds of interaction interface standardization design methods in the teaching and research of robot teaching interface design. These design methods not only can improve the interactive experience of teaching device, but also provide certain research foundation for the future design of teaching device design and design parameter database. In this paper, the design of the physical interface and the touch screen interface are better simplified and integrated. It provides reference value for the design, interaction, extensibility, and portability of the teaching robot interaction design of future industrial robots.

\section{ACKNOWLEDGMENT}

Xiamen University Tan Kah Kee College and VIBOT Industrial Robot Innovation Laboratory.

Support by: 1. Fujian Social Science Planning Project (NO.:FJ2016B100). 2. Research project of education teaching reform in Fujian province. (NO.:FBJG20170155). 3. Fujian Province Education and Scientific Program for Young and middle-aged teachers (NO.:JAT170840)

\section{REFERENCES}

[1] TIAN Tao, DENG Shuangcheng,YANG Zhaolan,ZHANG Ze,ZHENG Haiyang,WANG Fuli,ZHOU Tangkai. [J] New Technology \& New Process, 2015,(3)92-94.

[2] Li You- fu, Jisheng-xing.Design of User Interface of Robot' s Teaching Pendant Based on WinCE[J].Industrial Control Computer,2016,29(3):27-30.

[3] Hao Ninghui, Lu Xiaobo Critical Thinking on Tangible User Interface Design[J]. Zhuangshi,2014,2(48):34-37.

[4] COOPER A.The Road of Interaction Design,High-tech Products[M]. Beijing:Publishing House of Electronics Industry,2006.

[5] YE Dong-dong,LI Shi-guo.Hierarchy of Needs and Design Strategy in Interaction Design[J].Packaging Engineering,2013,34(8):75-78.

[6] ZHENG Kai-fang.Research on Graphical User Interface Design Based on the Human Consciousness[D].Changsha:Hunan University,2010. 